\title{
Reverses of Young and Heinz inequalities for positive linear operators
}

\author{
S Malekinejad ${ }^{1 *}$, S Talebi ${ }^{2}$ and AG Ghazanfari ${ }^{3}$
}

\section{"Correspondence: maleki60313@gmail.com \\ ${ }^{1}$ Department of Mathematics, Payame Noor University, P.O. Box 19395-3697, Tehran, Iran Full list of author information is available at the end of the article}

\section{Abstract}

Let $A, B$ be invertible positive operators on a Hilbert space $H$. We present some improved reverses of Young type inequalities, in particular,

$$
(1-v)^{2 v}(A \nabla B)+(1-v)^{2(1-v)} H_{2 v}(A, B) \geq 2(1-v)(A \sharp B)
$$

and

$$
(1-v)^{2 v} H_{2 v}(A, B)+(1-v)^{2(1-v)}(A \nabla B) \geq 2(1-v)(A \sharp B),
$$

where $0 \leq v \leq \frac{1}{2}$

We also give some new inequalities involving the Heinz mean for the Hilbert-Schmidt norm.

\section{Introduction}

Let $H$ be a Hilbert space and let $B_{h}(H)$ be the semi-space of all bounded linear self-adjoint operators on $H$. Further, let $B(H)$ and $B(H)^{+}$, respectively, denote the set of all bounded linear operators on a complex Hilbert space $H$ and set of all positive operators in $B_{h}(H)$. The set of all positive invertible operators is denoted by $B(H)^{++}$. For $A, B \in B(H)$, $A^{*}$ denotes the conjugate operator of $A$. An operator $A \in B(H)$ is positive, and we write $A \geq 0$, if $(A x, x) \geq 0$ for every vector $x \in H$. If $A$ and $B$ are self-adjoint operators, the order relation $A \geq B$ means, as usual, that $A-B$ is a positive operator. The theory of operator means for positive (bounded linear) operators on a Hilbert space was initiated by Ando and established by him and Kubo in connection with Lowners theory for the operator monotone functions [1].

An operator mean is a binary operation $\sigma$ defined on the set of strictly positive operators, if the following conditions hold:

(1) $A \leq C, B \leq D \Rightarrow A \sigma B \leq C \sigma D$.

(2) $A_{n} \downarrow A, B_{n} \downarrow B \Rightarrow A_{n} \sigma B_{n} \downarrow A \sigma B$.

(3) $T^{*}(A \sigma B) T \leq\left(T^{*} A T\right) \sigma\left(T^{*} B T\right)$ for $T \in B(H)$.

(4) $I \sigma I=I$.

(c) 2016 Malekinejad et al. This article is distributed under the terms of the Creative Commons Attribution 4.0 International License (http://creativecommons.org/licenses/by/4.0/), which permits unrestricted use, distribution, and reproduction in any medium, provided you give appropriate credit to the original author(s) and the source, provide a link to the Creative Commons license, and indicate if changes were made. 
In addition, $A \sigma B=A^{\frac{1}{2}} f\left(A^{\frac{-1}{2}} B A^{\frac{-1}{2}}\right) A^{\frac{1}{2}}$ for all strictly positive operators $A$ and $B$. The monotone function $f$ is called the representing function of $\sigma$. Let $A, B \in B(H)$ be two positive operators and $v \in[0,1]$, then the $v$-weighted arithmetic mean of $A$ and $B$, denoted by $A \nabla_{v} B$, is defined as $A \nabla_{v} B=(1-v) A+v B$. If $A$ is invertible, the $v$-geometric mean of $A$ and $B$, denoted by $A \sharp_{\nu} B$, is defined as $A \sharp_{\nu} B=A^{\frac{1}{2}}\left(A^{\frac{-1}{2}} B A^{\frac{-1}{2}}\right)^{\nu} A^{\frac{1}{2}}$. In addition, if both $A$ and $B$ are invertible, the $v$-harmonic mean of $A$ and $B$, denoted by $A{ }_{\nu} B$ is defined as $A !_{v} B=\left((1-v) A^{-1}+v B^{-1}\right)^{-1}$. For more details, see Kubo and Ando [1]. When $v=\frac{1}{2}$, we write $A \nabla B, A \sharp B, A ! B$ for brevity, respectively. The operator version of the Heinz means is denoted by

$$
H_{\nu}(A, B)=\frac{A \sharp_{\nu} B+A \sharp_{1-\nu} B}{2},
$$

where $A, B \in B(H)^{++}$, and $v \in[0,1]$. The operator version of the Heron means is denoted by

$$
F_{\alpha}(A, B)=(1-\alpha)(A \sharp B)+\alpha(A \nabla B)
$$

for $0 \leq \alpha \leq 1$. It is well known that if $A$ and $B$ are positive invertible operators, then

$$
A \nabla_{\nu} B \geq A \sharp_{\nu} B \geq A !_{\nu} B
$$

for $0<v<1$.

To obtain inequalities for bounded self-adjoint operators on Hilbert space, we shall use the following monotonicity property for operator functions:

If $X \in B_{h}(H)$ with a spectrum $\operatorname{Sp}(X)$ and $f, g$ are continuous real-valued functions on $\operatorname{Sp}(X)$, then

$$
f(t) \geq g(t), \quad t \in \operatorname{Sp}(X) \quad \Rightarrow \quad f(X) \geq g(X) .
$$

For more details as regards this property, the reader is referred to [2].

The classical Young inequality says that if $a, b \geq 0$ and $v \in[0,1]$, then

$$
a^{v} b^{1-v} \leq v a+(1-v) b
$$

with the equality if and only if $a=b$.

Zhao et al. [3] gave an inequality for the Heinz and Heron means as follows:

If $A$ and $B$ are two positive and invertible operators, then

$$
H_{\nu}(A, B) \leq F_{\alpha(v)}(A, B)
$$

for $v \in[0,1]$, where $\alpha(v)=1-4\left(v-v^{2}\right)$.

Kai in [4] gave the following Young type inequalities:

$$
v^{2} a+(1-v)^{2} b \geq v^{2}(\sqrt{a}-\sqrt{b})^{2}+v^{2 v} a^{v} b^{1-v}
$$


for $v \in\left[0, \frac{1}{2}\right]$. Recently, Burqan and Khandaqji [5] gave the following reverse of the scalar Young type inequality:

$$
v^{2} a+(1-v)^{2} b \leq(1-v)^{2}(\sqrt{a}-\sqrt{b})^{2}+a^{v}\left[(1-v)^{2} b\right]^{1-v}
$$

for $v \in\left[0, \frac{1}{2}\right]$. Also we have

$$
v^{2} a+(1-v)^{2} b \leq v^{2}(\sqrt{a}-\sqrt{b})^{2}+v^{2 v} a^{v} b^{1-v}
$$

for $v \in\left[\frac{1}{2}, 1\right]$.

\section{The results and discussion}

In this section, we present some converses of the Young inequality and give several refinements for matrices and operators.

\subsection{Reverses of scalar Young type inequalities}

First, we get reverses of the inequalities (1.4), (1.5), and (1.6).

Theorem 1 Let $a, b>0$ and $v \in\left[0, \frac{1}{2}\right]$. Then

$$
(1-v)^{2 v}[(1-2 v) a+2 v b]+a^{2 v}(1-v)^{2(1-v)} b^{1-2 v} \geq 2(1-v) \sqrt{a b}
$$

and

$$
(1-v)^{2(1-v)}[(2 v) a+(1-2 v) b]+a^{1-2 v}(1-v)^{2 v} b^{2 v} \geq 2(1-v) \sqrt{a b}
$$

Proof If $0 \leq v \leq \frac{1}{2}$, then by inequality (1.2), we have

$$
\begin{aligned}
(1- & v)^{2 v}[(1-2 v) a+2 v b]+a^{2 v}(1-v)^{2(1-v)} b^{1-2 v}-2(1-v) \sqrt{a b} \\
& \geq(1-v)^{2 v}\left[a^{1-2 v} b^{2 v}\right]+a^{2 v}(1-v)^{2(1-v)} b^{1-2 v}-2(1-v) \sqrt{a b} \\
& =a^{1-2 v}(1-v)^{2 v} b^{2 v}+a^{2 v}(1-v)^{2(1-v)} b^{1-2 v}-2(1-v) \sqrt{a b} \\
& =\left(a^{\frac{1-2 v}{2}}(1-v)^{v} b^{v}-a^{v}(1-v)^{(1-v)} b^{\frac{1-2 v}{2}}\right)^{2} \geq 0
\end{aligned}
$$

and similarly

$$
\begin{aligned}
(1- & v)^{2(1-v)}[(2 v) a+(1-2 v) b]+a^{1-2 v}(1-v)^{2 v} b^{2 v}-2(1-v) \sqrt{a b} \\
& \geq(1-v)^{2 v}\left[a^{1-2 v} b^{2 v}\right]+a^{2 v}(1-v)^{2(1-v)} b^{1-2 v}-2(1-v) \sqrt{a b} \\
& =\left(a^{\frac{1-2 v}{2}}(1-v)^{v} b^{v}-a^{v}(1-v)^{(1-v)} b^{\frac{1-2 v}{2}}\right)^{2} \geq 0 .
\end{aligned}
$$

Theorem 2 Let $a, b>0$ and $v \in\left[0, \frac{1}{2}\right]$. Then

$$
\begin{aligned}
& (1-v)^{2 v} a^{1-v} b^{v}+(1-v)^{2(1-v)}[v a+(1-v) b] \geq 2(1-v) \sqrt{a b}, \\
& (1-v)^{2 v}[(1-v) a+v b]+a^{v} b^{1-v}(1-v)^{2(1-v)} \geq 2(1-v) \sqrt{a b} .
\end{aligned}
$$


Proof If $0 \leq v \leq \frac{1}{2}$, then by inequality (1.2), we have

$$
\begin{gathered}
(1-v)^{2 v} a^{1-v} b^{v}+(1-v)^{2(1-v)}[v a+(1-v) b]-2(1-v) \sqrt{a b} \\
\geq(1-v)^{2 v} a^{1-v} b^{v}+(1-v)^{2(1-v)} a^{v} b^{1-v}-2(1-v) \sqrt{a b} \\
=(1-v)^{2 v} a^{1-v} b^{v}+a^{v}\left[(1-v)^{2} b\right]^{(1-v)}-2(1-v) \sqrt{a b} \\
=\left(a^{\frac{1-v}{2}}(1-v)^{v} b^{\frac{v}{2}}-a^{\frac{v}{2}}(1-v)^{(1-v)} b^{\frac{1-v}{2}}\right)^{2} \geq 0
\end{gathered}
$$

and similarly

$$
\begin{gathered}
(1-v)^{2 v}[(1-v) a+v b]+a^{v} b^{1-v}(1-v)^{2(1-v)}-2(1-v) \sqrt{a b} \\
\geq(1-v)^{2 v} a^{1-v} b^{v}+a^{v}\left[(1-v)^{2} b\right]^{(1-v)}-2(1-v) \sqrt{a b} \\
=\left(a^{\frac{1-v}{2}}(1-v)^{v} b^{\frac{v}{2}}-a^{\frac{v}{2}}(1-v)^{(1-v)} b^{\frac{1-v}{2}}\right)^{2} \geq 0 .
\end{gathered}
$$

Corollary 1 Let $a, b>0$ and $v \in\left[0, \frac{1}{2}\right]$. Then

$$
\begin{aligned}
& (1-v)^{2 v}\left(\frac{a+b}{2}\right)+(1-v)^{2(1-v)}\left(\frac{a^{2 v} b^{1-2 v}+a^{1-2 v} b^{2 v}}{2}\right) \\
& \geq 2(1-v)(\sqrt{a b}), \\
& (1-v)^{2(1-v)}\left(\frac{a+b}{2}\right)+(1-v)^{2 v}\left(\frac{a^{2 v} b^{1-2 v}+a^{1-2 v} b^{2 v}}{2}\right) \\
& \quad \geq 2(1-v)(\sqrt{a b}), \\
& (1-v)^{2 v}\left(\frac{a+b}{2}\right)+(1-v)^{2(1-v)}\left(\frac{a^{v} b^{1-v}+a^{1-v} b^{v}}{2}\right) \\
& \quad \geq 2(1-v)(\sqrt{a b}) .
\end{aligned}
$$

\subsection{Reverses of operator Young type inequalities}

We begin this section with the reverses of Young type inequalities for operators.

Theorem 3 Let $A, B \in B(H)^{++}$and $v \in\left[0, \frac{1}{2}\right]$. Then

$$
(1-v)^{2 v}(A \nabla B)+(1-v)^{2(1-v)} H_{2 v}(A, B) \geq 2(1-v)(A \sharp B)
$$

and

$$
(1-v)^{2 v} H_{2 v}(A, B)+(1-v)^{2(1-v)}(A \nabla B) \geq 2(1-v)(A \sharp B) .
$$

Proof If $v \in\left[0, \frac{1}{2}\right]$, the inequality (2.1), for $a=1, b>0$, becomes

$$
(1-v)^{2 v}[(1-2 v)+2 v b]+(1-v)^{2(1-v)} b^{1-2 v} \geq 2(1-v) \sqrt{b}
$$


The operator $X=A^{-\frac{1}{2}} B A^{-\frac{1}{2}}$ has a positive spectrum. According to rule (1.1), we can insert $A^{-\frac{1}{2}} B A^{-\frac{1}{2}}$ in the above inequality, i.e., we have

$$
\begin{aligned}
& (1-v)^{2 v}\left[(1-2 v)+2 v A^{-\frac{1}{2}} B A^{-\frac{1}{2}}\right]+(1-v)^{2(1-\nu)}\left(A^{-\frac{1}{2}} B A^{-\frac{1}{2}}\right)^{1-2 v} \\
& \quad \geq 2(1-v)\left(A^{-\frac{1}{2}} B A^{-\frac{1}{2}}\right)^{\frac{1}{2}} .
\end{aligned}
$$

Finally, if we multiply inequality (2.9) by $A^{\frac{1}{2}}$ on the left and right, we get

$$
(1-v)^{2 v}[(1-2 v) A+2 v B]+(1-v)^{2(1-v)}\left(A \sharp_{1-2 v} B\right) \geq 2(1-v)(A \sharp B) .
$$

By replacing $A$ by $B$ and $B$ by $A$, we have

$$
(1-v)^{2 v}[(1-2 v) B+2 v A]+(1-v)^{2(1-v)}\left(A \sharp_{2 v} B\right) \geq 2(1-v)(A \sharp B),
$$

and by the sum of (2.10) and (2.11), we have

$$
(1-v)^{2 v}(A \nabla B)+(1-v)^{2(1-v)} H_{2 v}(A, B) \geq 2(1-v)(A \sharp B) .
$$

Similarly, the inequality (2.2) implies that

$$
(1-v)^{2 v} H_{2 v}(A, B)+(1-v)^{2(1-v)}(A \nabla B) \geq 2(1-v)(A \sharp B) .
$$

Using the same strategy as in the proof of Theorem 3 and inequalities (2.3) and (2.4), we get the following theorems.

Theorem 4 Let $A, B \in B(H)^{++}$and $v \in\left[0, \frac{1}{2}\right]$. Then

$$
(1-v)^{2 v} H_{v}(A, B)+(1-v)^{2(1-v)}(A \nabla B) \geq 2(1-v)(A \sharp B) .
$$

Theorem 5 Let $A, B \in B(H)^{++}$and $v \in\left[0, \frac{1}{2}\right]$ Then

$$
(1-v)^{2(1-v)} H_{\nu}(A, B)+(1-v)^{2 v}(A \nabla B) \geq 2(1-v)(A \sharp B) .
$$

\subsection{Reverses of Young type inequalities for matrices}

In the following, let $M_{n}(C)$ be the space of all $n \times n$ complex matrices. For Hermitian matrices $A, B \in M_{n}(C)$, we write $A \geq 0$ if $A$ is positive semidefinite, $A>0$ if $A$ is positive definite, and $A \geq B$ if $A-B \geq 0$.

The Hilbert-Schmidt (or Frobenius) norm of $A=\left[a_{i j}\right] \in M_{n}(C)$ is denoted by $\|A\|_{2}=$ $\left(\sum_{j=1}^{n} s_{j}^{2}(A)\right)^{\frac{1}{2}}$, where $s_{1}(A) \geq s_{2}(A) \geq \cdots \geq s_{n}(A)$ are the singular values of $A$, which are the eigenvalues of the positive semidefinite matrix $|A|=\left(A A^{*}\right)^{\frac{1}{2}}$, arranged in decreasing order and repeated according to multiplicity. It is well known that the Hilbert-Schmidt norm is unitarily invariant.

For more information on matrix versions of the Young inequality (1.2) the reader is referred to [6]. In this section, we will discuss the reverse Heinz mean inequality for unitarily invariant norms. 
A matrix version of the inequality (1.3) is

$$
\left\|\frac{A^{v} X B^{1-v}+A^{1-v} X B^{v}}{2}\right\| \leq\left\|(1-\alpha(v)) A^{\frac{1}{2}} X B^{\frac{1}{2}}+\alpha(v)\left(\frac{A X+X B}{2}\right)\right\|,
$$

which was introduced by Bhatia [7].

The matrix version of the inequality

$$
a \sharp b \leq H_{v}(a, b) \leq a \nabla b,
$$

was proved by Bhatia and Davis [8], saying that if $0 \leq v \leq 1$, then

$$
\left\|A^{\frac{1}{2}} X B^{\frac{1}{2}}\right\| \leq\left\|\frac{A^{v} X B^{1-v}+A^{1-v} X B^{v}}{2}\right\| \leq\left\|\frac{A X+X B}{2}\right\| .
$$

The second part of the inequality (2.12) is known as the Heinz inequality. Let $0 \leq v \leq 1$, $r_{0}=\min \{v, 1-v\}$, Kittaneh [9] gave a refinement of the Heinz inequality as follows:

$$
\left\|\frac{A^{v} X B^{1-v}+A^{1-v} X B^{v}}{2}\right\| \leq 2 r_{0}\left\|A^{\frac{1}{2}} X B^{\frac{1}{2}}\right\|+\left(1-2 r_{0}\right)\left\|\frac{A X+X B}{2}\right\| .
$$

Meanwhile, Kittaneh and Manasarah [10] also obtained two refinements of the Heinz inequality for the Hilbert-Schmidt norm as follows:

$$
\begin{aligned}
& \left\|\frac{A^{v} X B^{1-v}+A^{1-v} X B^{v}}{2}\right\|_{2}^{2} \leq\left\|\frac{A X+X B}{2}\right\|_{2}^{2}-2 r_{0}\left\|\frac{A X-X B}{2}\right\|_{2}^{2}, \\
& \left\|\frac{A^{v} X B^{1-v}+A^{1-v} X B^{v}}{2}\right\|_{2} \leq\left\|\frac{A X+X B}{2}\right\|_{2}-r_{0}\left(\sqrt{\|A X\|_{2}}-\sqrt{\|X B\|_{2}}\right)^{2} .
\end{aligned}
$$

He et al. [11] proved that

$$
\left\|\frac{A^{v} X B^{1-v}+A^{1-v} X B^{v}}{2}\right\|^{2} \leq 2 r_{0}\left\|A^{\frac{1}{2}} X B^{\frac{1}{2}}\right\|^{2}+\left(1-2 r_{0}\right)\left\|\frac{A X+X B}{2}\right\|^{2} .
$$

It is weaker than the inequality (2.13) and it is equivalent to the inequality (2.14) for the Hilbert-Schmidt norm [11]. Zhan [12] proved that if $\frac{1}{4} \leq v \leq \frac{3}{4}$ and $-2<t \leq 2$, then

$$
\left\|\frac{A^{v} X B^{1-v}+A^{1-v} X B^{v}}{2}\right\| \leq \frac{1}{t+2}\left\|t A^{\frac{1}{2}} X B^{\frac{1}{2}}+A X+X B\right\| .
$$

It is also a refinement of the Heinz inequality for matrices. Zou [13] proved that if $0 \leq v \leq$ 1 , then

$$
\left\|\frac{A^{v} X B^{1-v}+A^{1-v} X B^{v}}{2}\right\|_{2}^{2} \leq\left\|\frac{A X+X B}{2}\right\|_{2}^{2}-a v(1-v)\left\|\frac{A X-X B}{2}\right\|_{2}^{2},
$$

which is an improvement of (2.14). Zou also, in [14], has proved another Heinz inequality for the Hilbert-Schmidt norm as follows. 
Theorem 6 Let $\alpha(v)=1-4\left(v-v^{2}\right)$ and $0 \leq v \leq 1$. Then

$$
\left\|\frac{A^{v} X B^{1-v}+A^{1-v} X B^{v}}{2}\right\|_{2} \leq\left\|(1-\alpha(v)) A^{\frac{1}{2}} X B^{\frac{1}{2}}+\alpha(v) \frac{A X+X B}{2}\right\|_{2} .
$$

Kittaneh and Manasarah in [6] have showed that if $A$ and $B$ are positive definite matrices, $X \in M_{n}$ and $v \in[0,1]$, then

$$
\begin{aligned}
& \|(1-v) A X+v X B\|_{2}^{2} \leq\left\|A^{1-v} X B^{v}\right\|_{2}^{2}+R^{2}\|A X-X B\|_{2}^{2}, \\
& \|A X+X B\|_{2}^{2} \leq\left\|A^{1-v} X B^{v}+A^{v} X B^{1-v}\right\|_{2}^{2}+2 R\|A X-X B\|_{2}^{2},
\end{aligned}
$$

where $R=\max \{v, 1-v\}$.

Bakherad and Moslehian [15] improved the Young inequality and obtained the following inequalities:

$$
\|A X+X B\|_{2}^{2}+2(v-1)\|A X-X B\|_{2}^{2} \leq\left\|A^{v} X B^{1-v}+A^{1-v} X B^{v}\right\|_{2}^{2}
$$

where $A$ and $B$ are positive definite matrices, $X \in M_{n}(C)$ and $v>1$.

Theorem 7 Let $A, B, X \in M_{n}$ such that $A$ and $B$ are positive definite; if $v \in\left[0, \frac{1}{2}\right]$, then

$$
\begin{aligned}
& \left\|(1-v)^{2(1-v)} \frac{A^{2 v} X B^{1-2 v}+A^{1-2 v} X B^{2 v}}{2}+(1-v)^{2 v} \frac{A X+X B}{2}\right\|_{2} \\
& \quad \geq 2(1-v)\left\|A^{\frac{1}{2}} X B^{\frac{1}{2}}\right\|_{2} .
\end{aligned}
$$

Proof Since $A$ and $B$ are positive semidefinite, it follows by the spectral theorem that there exist unitary matrices $U, V \in M_{n}$ such that

$$
A=U \Gamma_{1} U^{*} \quad \text { and } \quad B=V \Gamma_{2} V^{*}
$$

where

$$
\Gamma_{1}=\operatorname{diag}\left(\lambda_{1}, \ldots, \lambda_{n}\right), \quad \Gamma_{2}=\operatorname{diag}\left(\mu_{1}, \ldots, \mu_{n}\right), \quad \lambda_{i}, \mu_{i} \geq 0, i=1, \ldots, n
$$

Let

$$
Y=U^{*} X V=\left[y_{i j}\right]
$$

then

$$
\begin{aligned}
\frac{A^{v} X B^{1-v}+A^{1-v} X B^{v}}{2} & \\
= & \frac{\left(U \Gamma_{1} U^{*}\right)^{v} X\left(V \Gamma_{2} V^{*}\right)^{1-v}+\left(U \Gamma_{1} U^{*}\right)^{1-v} X\left(V \Gamma_{2} V^{*}\right)^{v}}{2} \\
= & \frac{\left(U \Gamma_{1}^{v} U^{*}\right) X\left(V \Gamma_{2}^{1-v} V^{*}\right)+\left(U \Gamma_{1}^{1-v} U^{*}\right) X\left(V \Gamma_{2}^{v} V^{*}\right)}{2}
\end{aligned}
$$




$$
\begin{aligned}
& =\frac{U \Gamma_{1}^{\nu}\left(U^{*} X V\right) \Gamma_{2}^{1-\nu} V^{*}+U \Gamma_{1}^{1-\nu}\left(U^{*} X V\right) \Gamma_{2}^{\nu} V^{*}}{2} \\
& =U\left(\frac{\Gamma_{1}^{v} Y \Gamma_{2}^{1-\nu}+\Gamma_{1}^{1-\nu} Y \Gamma_{2}^{\nu}}{2}\right) V^{*} .
\end{aligned}
$$

Therefore,

$$
\begin{aligned}
\left\|\frac{A^{v} X B^{1-v}+A^{1-v} X B^{v}}{2}\right\|_{2}^{2} & =\left\|\frac{\Gamma_{1}^{v} Y \Gamma_{2}^{1-v}+\Gamma_{1}^{1-v} Y \Gamma_{2}^{v}}{2}\right\|_{2}^{2} \\
& =\sum_{i, j=1}^{n}\left(\frac{\lambda_{i}^{v} \mu_{j}^{1-v}+\lambda_{i}^{1-v} \mu_{j}^{v}}{2}\right)^{2}\left|y_{i j}\right|^{2} .
\end{aligned}
$$

Similarly, we have

$$
\begin{aligned}
& \sum_{i, j=1}^{n}\left(\sqrt{\lambda_{i} \mu_{j}}\right)^{2}\left|y_{i j}\right|^{2}=\left\|A^{\frac{1}{2}} X B^{\frac{1}{2}}\right\|_{2}^{2}, \\
& \sum_{i, j=1}^{n}\left(\frac{\lambda_{i}+\mu_{j}}{2}\right)^{2}\left|y_{i j}\right|^{2}=\left\|\frac{A X+X B}{2}\right\|_{2}^{2} .
\end{aligned}
$$

It follows from the inequality (2.5) that

$$
\begin{aligned}
\|(1 & -v)^{2(1-v)} \frac{A^{2 v} X B^{1-2 v}+A^{1-2 v} X B^{2 v}}{2}+(1-v)^{2 v} \frac{A X+X B}{2} \|_{2}^{2} \\
& =\sum_{i, j=1}^{n}\left((1-v)^{2(1-v)} \frac{\lambda_{i}^{2 v} \mu_{j}^{1-2 v}+\lambda_{i}^{1-2 v} \mu_{j}^{2 v}}{2}+(1-v)^{2 v} \frac{\lambda_{i}+\mu_{j}}{2}\right)^{2}\left|y_{i j}\right|^{2} \\
& \geq \sum_{i, j=1}^{n}\left(2(1-v) \sqrt{\lambda_{i} \mu_{j}}\right)^{2}\left|y_{i j}\right|^{2}=(2(1-v))^{2}\left\|A^{\frac{1}{2}} X B^{\frac{1}{2}}\right\|_{2}^{2} .
\end{aligned}
$$

Using the same strategy as in the proof of Theorem 7 and inequalities (2.6) and (2.7), we get the following theorems.

Theorem 8 Let $A, B, X \in M_{n}$ such that $A$ and $B$ are positive definite if $v \in\left[0, \frac{1}{2}\right]$, then

$$
\begin{aligned}
& \left\|(1-v)^{2 v} \frac{A^{2 v} X B^{1-2 v}+A^{1-2 v} X B^{2 v}}{2}+(1-v)^{2(1-v)} \frac{A X+X B}{2}\right\|_{2} \\
& \quad \geq\left\|2(1-v) A^{\frac{1}{2}} X B^{\frac{1}{2}}\right\|_{2} .
\end{aligned}
$$

Theorem 9 Let $A, B, X \in M_{n}$ such that $A$ and $B$ are positive definite if $v \in\left[0, \frac{1}{2}\right]$, then

$$
\begin{aligned}
& \left\|(1-v)^{2(1-v)} \frac{A^{v} X B^{1-v}+A^{1-v} X B^{v}}{2}+(1-2 v)^{2 v} \frac{A X+X B}{2}\right\|_{2} \\
& \quad \geq\left\|2(1-v) A^{\frac{1}{2}} X B^{\frac{1}{2}}\right\|_{2} .
\end{aligned}
$$




\section{Conclusions}

In the present paper we got reverses of the scalar Young type inequality and using them we obtained the reverses of Young type inequalities for operators. Then we considered the reverse Heinz mean inequality for unitarily invariant norms and established the following inequality and several related results:

$$
\begin{aligned}
& \left\|(1-v)^{2(1-v)} \frac{A^{2 v} X B^{1-2 v}+A^{1-2 v} X B^{2 v}}{2}+(1-v)^{2 v} \frac{A X+X B}{2}\right\|_{2} \\
& \quad \geq 2(1-v)\left\|A^{\frac{1}{2}} X B^{\frac{1}{2}}\right\|_{2},
\end{aligned}
$$

where $A, B, X \in M_{n}$ such that $A$ and $B$ are positive definite and $v \in\left[0, \frac{1}{2}\right]$.

\section{Competing interests}

The authors declare that they have no competing interests.

\section{Authors' contributions}

All authors conceived of the study, and participated in the design and coordination. All authors read and approved the final manuscript.

\section{Author details}

'Department of Mathematics, Payame Noor University, P.O. Box 19395-3697, Tehran, Iran. ${ }^{2}$ Department of Mathematics, Payame Noor University, P.O. Box 91735-433, Mashhad, Iran. ${ }^{3}$ Department of Mathematics, Lorestan University, P.O. Box 465, Khoramabad, Iran.

\section{Acknowledgements}

The authors wish to express their hearty thanks to the referees for their valuable comments, detailed corrections, and suggestions for revising the manuscript.

Received: 10 November 2015 Accepted: 14 January 2016 Published online: 29 January 2016

\section{References}

1. Kubo, F, Ando, T: Means of positive linear operators. Math. Ann. 246, 205-224 (1980)

2. Pečarić, JE, Furuta, T, Mićić Hot, J, Seo, Y: Mond-Pečarić Method in Operator Inequalities. Element, Zagreb (2005)

3. Zhao, J, Wu, J, Cao, H, Liao, W: Operator inequalities involving the arithmetic, geometric, Heinz and Heron means. J. Math. Inequal. 8(4), 747-756 (2014)

4. Kai, H: Young type inequalities for matrices. J. East China Norm. Univ. Natur. Sci. Ed. 4, 12-17 (2012)

5. Burqan, A, Khandaqji, M: Reverses of Young type inequalities. J. Math. Inequal. 9(1), 113-120 (2015)

6. Kittaneh, F, Manasarah, Y: Reverse Young and Heinz inequalities for matrices. Linear Multilinear Algebra 59, 1031-1037 (2011)

7. Bhatia, R: Interpolating the arithmetic-geometric mean inequality and its operator version. Linear Algebra Appl. 413, 355-363 (2006)

8. Bhatia, R, Davis, C: More matrix forms of the arithmetic-geometric mean inequality. SIAM J. Matrix Anal. Appl. 14, $132-136(1993)$

9. Kittaneh, F: On the convexity of the Heinz means. Integral Equ. Oper. Theory 68, 519-527 (2010)

10. Kittaneh, F, Manasarah, Y: Improved Young and Heinz inequalities for matrices. J. Math. Anal. Appl. 361, 262-269 (2010)

11. He, C, Zou, L, Qaisar, S: On improved arithmetic-geometric mean and Heinz inequalities for matrices. J. Math. Inequal. 6, 453-459 (2012)

12. Zhan, X: Inequalities for unitarily invariant norms. SIAM J. Matrix Anal. Appl. 20, 466-470 (1998)

13. Zou, L: On some matrix inequalities. Acta Math. Sin. Chin. Ser. 55, 715-720 (2012)

14. Zou, L: Inequalities related to Heinz and Heron means. J. Math. Inequal. 7(3), 389-397 (2013)

15. Bakherad, M, Moslehian, MS: Reverses and variations of Heinz inequality. Linear Multilinear Algebra (2014). doi:10.1080/03081087.2014.880433 